\title{
Relationship between classical Hodgkin's lymphoma and diffuse large B-cell lymphoma: Separate entity or biologic continuum
}

\author{
Salvia Jain, Bruce Raphael, Kent P Friedman \\ Xiaowei Chen, Sherif Ibrahim
}

\begin{abstract}
Introduction: Cases of patients with nonHodgkin's lymphoma observed after therapy for Hodgkin lymphoma have been reported. However, occurrence of non-Hodgkin's lymphomas in such settings at a previously uninvolved site is extremely rare. The mechanism behind this remains obscure thereby presenting diagnostic and therapeutic dilemma for the treating physician. Case Report: We report a case of a 62-year-old woman who developed a large B-cell nonHodgkin's lymphoma at a different anatomic site six months after receiving high dose chemotherapy and autologous stem cell transplantation for her relapsed Hodgkin lymphoma. We treated her with conventional combination chemoimmunotherapy following which she achieved durable complete remission. Conclusion: This case highlights the intriguing relationship between classical Hodgkin lymphoma and B-cell non- Hodgkin's
\end{abstract}

Salvia Jain ${ }^{1}$, Bruce Raphael ${ }^{2}$, Kent P Friedman ${ }^{3}$, Xiaowei Chen, Sherif Ibrahim ${ }^{4}$

Affiliations: ${ }^{1}$ Division of Hematology and Medical Oncology, NYU Cancer Institute, New York, NY, USA; ${ }^{2}$ Division of Radiology, NYU Langone Medical Center, New York, NY, USA; ${ }^{3}$ Division of Pathology, Columbia University Medical Center, New York, NY, USA; ${ }^{4}$ Division of Hematopathology, NYU Cancer Institute, New York, NY, USA.

Corresponding Author: Salvia Jain, NYU Langone Medical Center, 550 First Avenue, New York, New York USA-10016; Phone: 001-650-224-0183; Fax: 001-212562-1117; Email: salvia.jain@nyumc.org

Received: 08 March 2011

Accepted: 15 May 2011

Published: 31 May 2011 lymphoma with respect to pathogenesis and its impact on therapeutic decisions and outcomes in such contentious cases which a clinician must face.

Keywords: Classical Hodgkin lymphoma, Large B-cell lymphoma, Chemoimmunotherapy, Bcell gene rearrangement

$* * * * * * * * *$

Jain S, Raphael B, Friedman KP, Chen X, Ibrahim S. Relationship between classical Hodgkin's lymphoma and diffuse large B-cell lymphoma: Separate entity or biologic continuum. International Journal of Case Reports and Images 2011;2(5):13-20.

$* * * * * * * * *$

doi:10.5348/ijcri-2011-05-33-CR-3

\section{INTRODUCTION}

While Hodgkin's lymphoma and non-Hodgkin's lymphoma are considered distinct entities, they represent a continuous spectrum of malignant transformation of B-cells. Cases at the interface such as B-cell lymphoma, unclassifiable, with features intermediate between diffuse large B-cell lymphoma and classical Hodgkin's lymphoma, also otherwise called as "grey zone lymphoma" present diagnostic and therapeutic challenges. Additionally the presence of both Hodgkin's lymphoma (HL) and non-Hodgkin's lymphoma (NHL) either concurrently (composite lymphoma) or subsequently present the clinician with the dilemma of one tumor differentiating in two directions or two different tumors. We report a case of a 62-year-woman with classical Hodgkin's lymphoma who subsequently developed a large B-cell nonHodgkin's lymphoma at a different anatomic site 
within two years after initial disease and six months after receiving high dose chemotherapy and autologous stem cell transplantation for her relapsed Hodgkin's lymphoma. She was subsequently treated with conventional combination chemo-immunotherapy (REPOCH) and responded with a near complete remission. We describe the clinico-pathologic and radiological features of this unique case which raises the question whether the two B-cell tumors arise by independent mechanisms from different precursors or have a common cell of origin that undergoes transformation over time and review the literature regarding treatment and outcome of such lymphomas.

\section{CASE REPORT}

A-62-year old woman was referred with recent finding of abdominal lymphadenopathy. She had presented to an outside institution with Hematuria. A CT scan of the abdomen and pelvis with contrast demonstrated lymphadenopathy in the retroperitoneal (RP) area leading to left hydronephrosis (Figure 1A). She also complained of B symptoms but her physical examination did not reveal any palpable lymphadenopathy or organomegaly and her laboratory results including serum LDH level were unremarkable. Management at our institution included placement of a left ureteral stent to relieve the obstruction and excisional biopsy of the retroperitoneal lymph node to characterize the underlying etiology.

The biopsy showed alteration of the nodal architecture by a nodular proliferation with thick, fibrotic septa. Marked nodal capsular fibrosis was also noted. The proliferation contained mixed inflammatory cells of small lymphocytes and histiocytes with scattered large atypical cells consistent with Reed-Sternberg (RS) cells and some variants (Figure 2A). Immunohistochemical (IHC) staining was positive for $\mathrm{CD}_{3} \mathrm{O}$ in the malignant Hodgkin's and Reed Sternberg (HRS) cells (Fig. 2B). The RS cells did not demonstrate CD15 and CD45 staining but showed variable $\mathrm{CD} 20$ staining (Figure $2 \mathrm{C}$ ). The biopsy was also reviewed at the National Cancer institute (NCI) and felt to be consistent with the diagnosis of classical Hodgkin lymphoma, nodular sclerosing subtype. Molecular studies were performed for T-cell receptor and immunoglobulin light and heavy chain gene rearrangement but did not demonstrate clonal rearrangement of $\mathrm{B}$ or T-cells.

A staging PET scan confirmed the extensive retroperitoneal and bilateral pelvic lymphadenopathy measuring $9 \times 5.4 \mathrm{~cm}$ with mean standard uptake values ranging between 3 and 5.7. Thus the patient was diagnosed with stage IIB classical Hodgkin lymphoma with unfavorable NCCN factors (ESR $>50$, > 3 lymphoid regions, B-symptoms). Hence she was treated with six cycles of ABVD (adriamycin, bleomycin, vinblastine and dacarbazine) and achieved complete remission.
She relapsed within three months of completing initial chemotherapy and after pathological confirmation received salvage chemotherapy with two cycles of ICE (ifosfamide, carboplatinum and etoposide) followed by autologous transplantation after conditioning with BEAM (carmustine, etoposide, cytarabine and melphalan). She remained in remission for nine months but developed local recurrence of Hodgkin lymphoma in the pelvis for which pelvic irradiation (3960 cGy in 22 fractions) was administered.

Surveillance PET scan at six months after finishing radiation showed decrease in the size and intensity of pelvic lymphadenopathy but demonstrated a new hyermetabolic enhancing mass approximately $4.9 \times 2.8$ $\mathrm{cm}$ in size in the vaginal cuff (Figure 1B) with new retroperitoneal, mesenteric and left inguinal lymphadenopathy. The biopsy of the vaginal mass showed sheets of large atypical cells with round to oval nuclei, finely granular chromatin and central large nucleoli (Figure 2D). IHC staining revealed bright expression of CD2O (Fig.2F), dim expression of CD3O (Figure 2E), CD79, PAX-5, OCT-2, PU.1, Bcl-2 but did not show expression of EBV RNA (EBER) by in situ hybridization in the tumor cells. B-cell gene rearrangements were investigated using IGK Gene Clonality Assay from IVS (repeated twice) and with Jk -home brew. For the heavy chain: IGH (FR2 and FR3) we used home brew (lab developed test, LDT).We checked for both immunoglobulin heavy and light chains gene rearrangements. Molecular studies demonstrated kappa light chain clonal rearrangement in the B-cells (Figure 2G).This was felt to be consistent with the diagnosis of diffuse large B-cell lymphoma (DLBCL) with CD30 expression and once again was confirmed at the NCI.

Staging confirmed stage IV DLBCL with high IPI score. Given poor response of transformed large cell lymphomas to conventional chemotherapy regimens, our patient opted to go on an investigational protocol as a result of which she received Pralatrexate (PDX, 10propargyl 10-deazaaminopterin), a novel anti-folate drug which has been approved for treatment of relapsed or refractory peripheral T-cell lymphoma.

She was found to have progression of disease at the end of two cycles and hence her regimen was changed to an anthracycline based standard immunochemotherapy combination: R-EPOCH (rituxan, etoposide, prednisone, vincristine, cyclophosphamide and doxorubicin) of which she has received four cycles so far followed by near complete resolution of her large B-cell lymphoma (Figure 1C). We are contemplating administration of a total of six cycles of R-EPOCH and possibility of reduced intensity allogeneic transplantation while in remission to consolidate the favorable response.

\section{DISCUSSION}

We believe that the presence of persistent CD30 

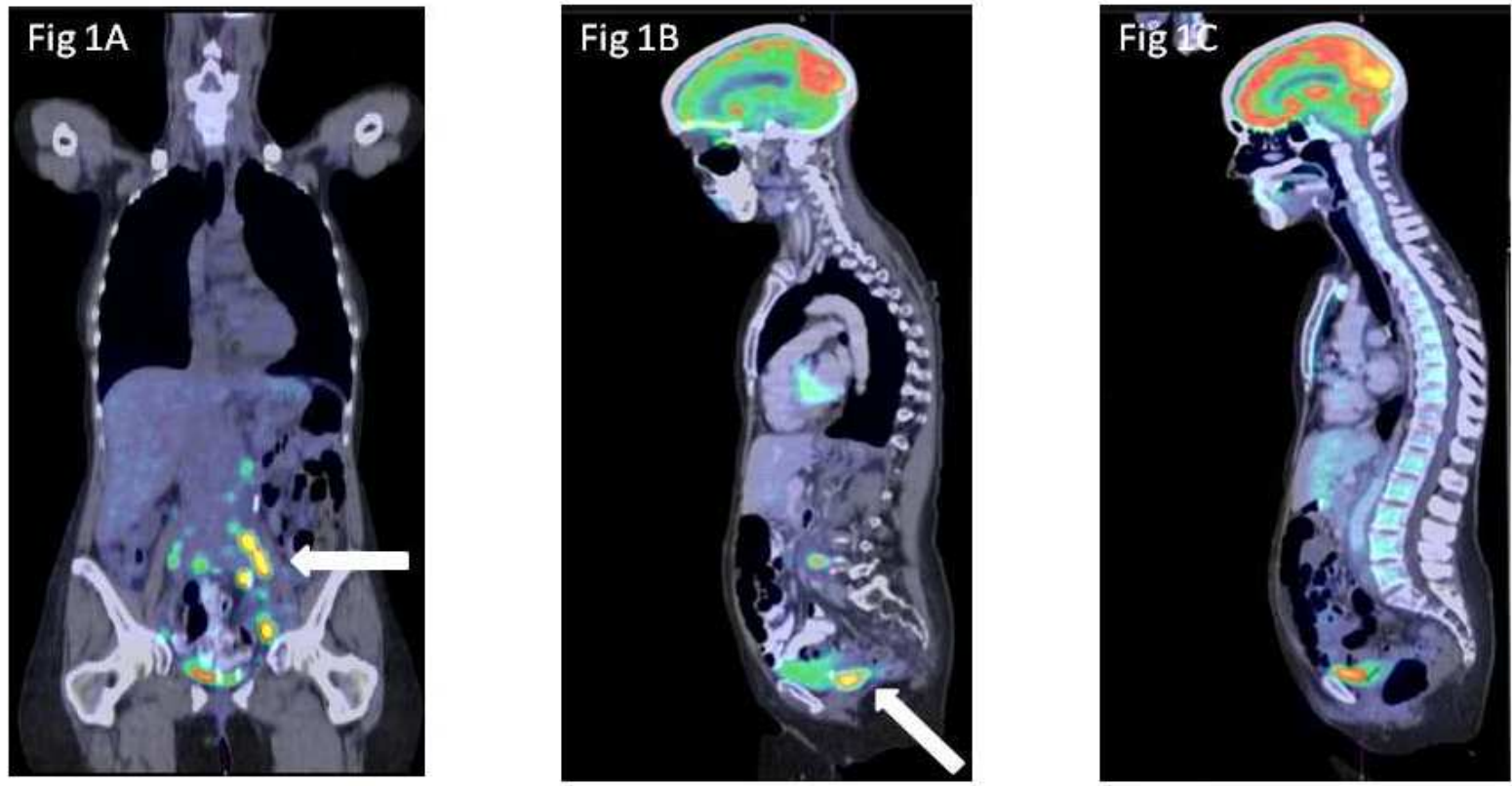

Figure1: A) Initial whole body FDG PET/CT scan with arrow demonstrating retroperitoneal and pelvic lymphadenopathy which are hypermetabolic with mean standard uptake value of this activity ranging between $3-5.7$; B) Second whole body FDG PET/CT scan (6 months after finishing pelvic radiation for relapse of Hodgkin's lymphoma) with arrow demonstrating a new 4.8x2.6 cm hypermetabolic vaginal mass with mean standard uptake value ranging between $7.2-11.5$; C) Third whole body FDG PET /CT scan (3 weeks after finishing 4 cycles of chemo-immunotherapy with R-EPOCH) demonstrating complete resolution of the hypermetabolic vaginal mass. Anterior uptake is secondary to normal bladder activity (FDG excretion by the kidneys).

and increased CD2O expression in the vaginal mass biopsy in our patient with a previous history of $\mathrm{cHL}$ raises a number of questions regarding the pathogenic interrelationship and treatment of such lymphomas. Observations from small number of case reports suggest an inherent tendency of nodular lymphocytepredominant Hodgkin's lymphoma (non-classical NLPHD) to develop DLBCL either concurrently or subsequently [1-6] with a wide range in the incidence rates (0.6-9.8\%) across case series over 20 years of follow up. It is now felt that this entity is really a form of follicular lymphoma. There are also case reports of the two diseases presenting together, commonly known as "composite lymphoma" [7-9]. However, little literature exists on the association between cHL and NHL because of the rarity of this condition. The reported incidence of NHL in large series of HL survivors has ranged from $0.9 \%$ at an average follow up of 6.7 years to $1.6 \%$ at 15 years [10-12]. Diffuse large B-cell lymphoma was the most common histology reported in these studies accounting for 78 percent of the cases. The largest experience comes from the International Database on Hodgkin Lymphoma, which evaluated the course of 12,411 patients with an average follow up of 6.7 years [10]. One hundred and six cases of NHL were observed with a continuous increase in relative risk with a follow up in males (from 28.2 during the five to nine year period, to 120 during the 15 to 19 year period). In females, the risk increase was confined to the first 15 years, with a peak relative risk of 45.4 during the five to nine year observation period.

While the pathogenesis of NHL's following Hodgkin lymphoma remains unknown some authors have speculated that they both originate from a common Bcell precursor, probably a germinal center B-cell and share some common and some distinct molecular transforming events [13-15]. It is sometimes possible to demonstrate the common cell of origin with careful molecular studies. With the help of polymerase chain reaction amplification we were able to identify and characterize clonal B cells by demonstrating presence of $\mathrm{J}_{\mathrm{k}}$ gene (joining region gene of Kappa light chain) rearrangement in the DLBCL tumor. We then generated clone-specific primers which could enable us to pick-up the same rearrangement in the HRS cells if the HRS cells and DLBCL tumor cells shared the same immunoglobulin light chain rearrangement which could provide valuable hint towards their common origin and thereby insight into the biology of such rare lymphomas. Unfortunately in our case the negative molecular studies for B-cell clonalilty i.e. absence of $\mathrm{J}_{\mathrm{K}}$ gene rearrangement in the cHL tumor sample were not helpful in establishing this possibly common malignant B-cell precursor. This is not uncommon and we believe raises the possibility of the two B-cell tumors arising from different precursors. Given 

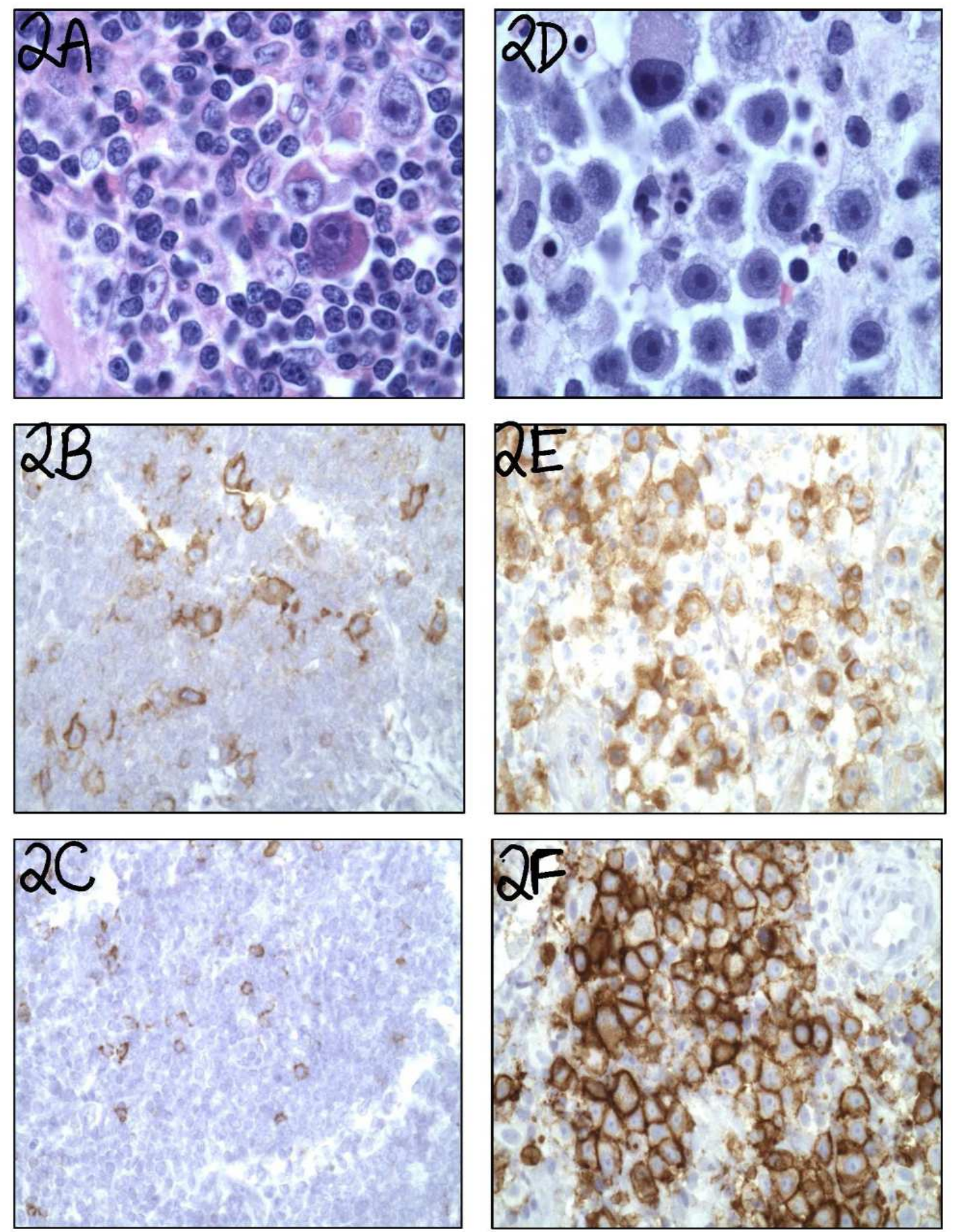

Figure2: A) Initial retroperitoneal lymph node biopsy (H\&E stain, oil immersion). It exhibits mixed inflammatory cells, lymphocytes and histiocytes. There are scattered large atypical cells, consistent with Reed-Sternberg cells with bizarre nuclear features; B) Initial retroperitoneal lymph node biopsy (CD30 staining, 40X,). It exhibits strong expression of CD30 in malignant HRS cells; C) Initial retroperitoneal lymph node biopsy (CD20 staining, 40X,). It exhibits few HRS cells that demonstrate weak expression of CD2O; D) Vaginal mass biopsy (H\&E stain, oil immersion). It exhibits diffuse lymphocytic infiltrate comprising of sheets of large lymphocytes with prominent eosinophilic nucleoli; E) Vaginal mass biopsy (CD30 staining, 40X,). It exhibits weak CD30 expression in most of the large neoplastic lymphocytes; F) Vaginal mass biopsy (CD20 staining, 4OX,). It exhibits sheets of large neoplastic lymphocytes with intense CD20 expression. 


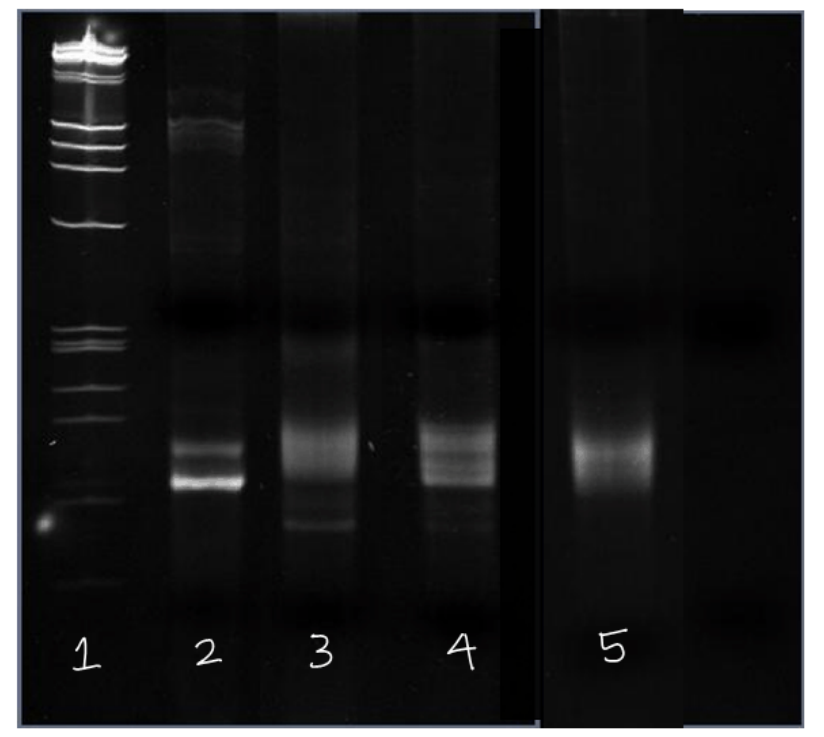

Figure 3: Polymerase chain reaction (PCR) for $\mathrm{J}_{\mathrm{k}}$ gene (joining region gene of Kappa light chain) rearrangement in cHL and DLBCL tumors; Lane $\mathbf{1}$ is the ladder; Lane 2 is the positive control for the $\mathrm{J}_{\mathrm{k}}$ gene rearrangement, is the negative control for the $J_{\mathrm{k}}$ gene rearrangement; Lane 4 is the diffuse large B-cell lymphoma sample showing a distinct band thereby confirming the presence of $\mathrm{J}_{\mathrm{k}}$ gene rearrangement which is a marker of clonal B cells; Lane 5 is the classical Hodgkin lymphoma sample showing a polyclonal pattern in the region of $J_{k}$ gene rearrangement thereby demonstrating absence of clonal B cells.

expression of CD2O on the HRS cells in the initial biopsy, another relevant question is whether the patient should have been diagnosed with B-cell lymphoma, unclassifiable, with features intermediate between diffuse large B-cell lymphoma and classical Hodgkin's lymphoma also called as "grey zone lymphoma" per the 2008 WHO classification for mature B-cell lymphomas. The majority of HRS cells in the first biopsy showed no expression of CD45 and CD79a but over-expression of CD30 with variable CD20 staining. The neoplastic cells of grey zone lymphomas typically express CD45. CD20 and CD79a are also frequently positive, and may be strongly expressed on the majority of the tumor cells. Because of the absence of strong and uniform expression of CD20, CD79a and CD45 on the tumor cells that favor the diagnosis of grey zone lymphoma, we believe that the immunohistochemical (IHC) characteristics of the first biopsy support the diagnosis of cHL and exclude the possibility of grey zone lymphoma although variants of each category cannot be entirely ruled out. Sometimes the neoplastic HRS cells of cHL do express $\mathrm{CD} 20$ with a reported frequency ranging from less than $5 \%$ to more than $50 \%$ of tumors [16-19] corroborating our hypothesis. However the prognostic significance of CD2O expression in cHL remains controversial [20-
23].

There is little agreement on definitive risk factors for NHL in patients treated for HL. It is possible that the differences among studies represent differences in the patient populations examined. It is also possible that pathologic misclassification may confound results, with recurrent HL being misdiagnosed as NHL or NHL being initially diagnosed as HL. Only a few studies have included expert review of the original tissue diagnosis as part of the study [11, 24-25]. With improvement in histopathologic techniques the risk of NHL appears to have declined over the past decade. A key question that is raised in such cases is whether the development of the subsequent large cell lymphoma was triggered by the chemotherapy, radiotherapy or combination regimens used for treating the $\mathrm{cHL}$ and for conditioning for autotransplantation. Most of the cytotoxic agents and radiation our patient received are capable of generating single and double stranded DNA breaks (DSB). Germinal Center (GC) tumors such as cHL and some other B-cell lymphomas seem to arise because of aberrations during B-cell development processes such as somatic hypermutation, class switch recombination and may be VDJ recombination, a major feature of which is DSB. Typically secondary NHL's develop after the first five years of initial therapy of primary cancer as opposed to our patient who developed NHL within two years of initial therapy for her HL. Hence based on clinical history, morphologic pattern and immunophenotypic studies we believe that the development of the NHL in this case is consistent with a transforming event between cHL and DLBCL or a biologic evolution of a malignant B-cell clone rather than a secondary malignancy. Whether cytotoxic agents and radiation can accelerate the transforming molecular mechanisms and contribute to biological aggressiveness remains to be ascertained. A large International Database Study included 106 patient who developed NHL following a prior malignancy; combined modality treatment and any type of treatment for relapse were associated with increased risk for NHL with relative risks of 2.2 to 4.5 [24]. However, other reports have found no apparent differences in increased risk between patients treated with chemotherapy alone, radiotherapy alone, or a combination modality [12, 24, 26-27]. There are also conflicting data on the importance of host factors such as gender and age on the risk of NHL. Some studies noted no relationship between these factors and NHL [28], while the International Database and report from the Netherlands Cancer Institute found that increasing age over 30 and to a lesser degree, male sex were risk factors $[10,25]$. In addition, HL may be associated with a general state of immunosuppression [29-30], which can predispose to the development of NHL as in transplant recipients. Support for this hypothesis comes from a detailed study of 14 patients who developed NHL within a median of 136 months from the diagnosis of HL [30]. The high prevalence of 
extranodal sites, histology of either intermediate or high grade lymphoma, and immunophenotypic findings typical of NHL of B-cell lineage patients were similar to NHL arising in immunosuppressed patients.

Little literature exists regarding the outcome of DLBCL after treatment of cHL. Initial studies reported poor outcomes [31]. However, later studies suggest that some of these patients may be curable with aggressive multi-agent chemotherapy [3, 4, 10, 11, 26, 30]. Of the 106 patients with secondary NHL in the International Database on HL (diagnosed between 1960 and 1987), 45 were alive at the time of report with a five year probability of survival of 30 percent. To investigate the course of disease for secondary NHL, Rueffer et al. retrospectively analyzed patients treated within clinical trials of the German Hodgkin's Lymphoma Study Group (GHSG) from 1981 to 1998 [11]. At first diagnosis of HL, the pathologists rejected 114 (2.1\%) of 5,520 cases initially diagnosed as HL and rediagnosed them as primary NHL. Fifty-two (0.9\%) of the remaining 5,406 patients developed a secondary NHL. One patient was excluded from further analyses because of insufficient documentation. Six patients had no further therapy because of patient refusal $(n=$ 1) or rapidly progressive disease $(n=5)$. For the remaining 45 patients, overall response rate was $43 \%$ (36\% complete response and $7 \%$ partial response). The actuarial 2-year freedom from treatment failure (FFTF) and overall survival (OS) for all patients was $24 \%$ and $30 \%$, respectively, and for patients with diffuse large-cell lymphoma, it was $28 \%$ and $35 \%$, respectively. Time of occurrence of secondary NHL after first diagnosis of HD and variables employed in the age-adjusted International Prognostic Factor Index (IPFI) significantly influenced treatment outcome. A retrospective analysis of patients in the Surveillance, Epidemiology and End Results (SEER) program database treated from 2000 to 2004 reported five and ten year survival rates for patients with secondary NHL of 54 and 41 percent respectively [32]. Despite relative worse prognosis compared with those with no prior malignancy, this data suggested a strong improvement in the outcomes for NHL patients with prior malignancy between 1990-1994 (five year survival rate of 38 percent and 10 year survival rate of $24 \%$ ). Until prospective long term follow up data from welldesigned randomized trials comparing outcome of de novo DLBCL with secondary DLBCL is available to guide therapeutic decision, we believe that patients like the one in our case report should be treated with multiagent chemotherapy regimens and enrolled in clinical trials when they fail established aggressive protocols.

Another clinical rule illustrated by this case that cannot be overemphasized is the importance of rebiopsy at disease progression or relapse specifically at an unusual previously uninvolved anatomical site. This is crucial to avoid misdiagnosis and allows effective management of this rare entity especially when whole body FDG PET/CT scan is becoming the standard of care in the follow up of cHL patients.

\section{CONCLUSION}

While it was difficult for us to establish that the initial classical Hodgkin lymphoma and subsequent development of large B-cell non-Hodgkin's lymphoma originated from the same malignant B-cell clone either synchronously or metachronously, an important point, this case has been thought provoking and educational at various stages of its evolution. We believe that this case brings to light several key issues in such challenging series of patients: first, it should not be presumed that the outcome of secondary large B-cell lymphomas with standard chemoimmunotherapy regimens is inferior or at least different than denovo cases until prospective randomized data from welldesigned clinical trials validates this conception. Second, while it is tempting to think that when patient's relapse from a hematologic malignancy, it is likely that this is the recurrence of the primary disease either due to residual resistant disease or further clonal evolution, this case highlights the importance of reconfirming tissue diagnosis at relapse or progression of the primary hematologic malignancy. Third, patients with Hodgkin lymphomas are always at risk of developing secondary malignancies of various phenotypes and hence active surveillance and close vigilance needs to be exercised both by the patient and by the clinician.

$* * * * * * * * *$

\section{Author Contributions}

Salvia Jain - Conception and design, Acquisition of data, Analysis and interpretation of data, Drafting the article, Critical revision of the article, Final approval of the version to be published

Bruce Raphael - Analysis and interpretation of data, Drafting the article, Critical revision of the article, Final approval of the version to be published

\section{Kent Friedman}

Group 1 - Conception and design, Acquisition of data, Analysis and interpretation of data, Critical revision of the article, Final approval of the version to be published

\section{Xiaowei Chen}

Group 1 - Conception and design, Acquisition of data, Analysis and interpretation of data, Critical revision of the article, Final approval of the version to be published

Sherif Ibrahim - Analysis and interpretation of data, Drafting the article, Critical revision of the article, Final approval of the version to be published

\section{Guarantor}

The corresponding author is the guarantor of submission. 


\section{Conflict of Interest}

The authors declare no conflict of interest with an institution or product mentioned in the manuscript.

\section{Copyright}

(C) Salvia Jain et al. 2011; This article is distributed under the terms of Creative Commons attribution 3.0 License which permits unrestricted use, distribution and reproduction in any means provided the original authors and original publisher are properly credited. (Please see www.ijcasereportsandimages.com /copyright-policy.php for more information.)

\section{REFERENCES}

1. Krikorian JG, Burke JS, Rosenberg SA, Kaplan HS et al. Occurrenceof non-Hodgkin's lymphoma after therapy for Hodgkin's disease. N Engl J Med 1979; 300:452-458.

2. Bennett MH, MacLennan KA, Vaughan $\mathrm{G}$ et al. Non-Hodgkin'slymphoma arising in patients treated for Hodgkin's disease in the BLNI: A20-year experience - British National Lymphoma Investigation. Annals of Oncology 1991 Supplement 2 to Volume 2:83-92.

3. Huang JZ,Weisenburger DD, Vose JM, et al. Diffuselarge B-cell Lymphoma arising in a nodular lymphocyte predominant Hodgkin Lymphoma, a report of 21 cases from the Nebraska Lymphoma Study group. Leukemia and Lymphoma 2003;44 (11):1903-1910.

4. Mansour MA, Connors JM,Gascoyne RD et al.Transformation to aggressive lymphoma in nodular lymphocyte predominant Hodgkin's lymphoma. JClinOncol 2010;28(5):793-799.

5. Armitage JO, Dick FR, Goeken JA, et al.Second lymphoid malignant neoplasms occurring in patientstreated for Hodgkin's disease. Arch Internal Med 1983;143:445-50.

6. Jaffe ES, Zarate-Osorno A, Kingman DW, et al.The inter-relationship between Hodgkin's disease and non-Hodgkin's lymphomas.Ann Oncol 1994 (suppl);5:7-11

7. Horschowski N, Sainty D, Favre R, et al. Composite Lymphoma. Review of the literature. Ann AnatPathol (Paris) 1980;25(4):307-315

8. Gonzales CL. Medeiros U. Jaffe ES: Composite lymphoma. Aclinico-pathologic analysis of nine patients with Hodgkin's diseaseand B-cell nonHodgkin's lymphoma. Am J ClinPathol 1991;96:8189.

9. Kim H. Composite lymphoma and related disorders. Am J ClinPathol 1993;99(4):445-451.

10. Henry-Amar M. Second cancer after the treatment for Hodgkin's disease: a report from the International Database on Hodgkin's Disease. Ann Oncol 1992;3 Suppl 4:117.

11. Rueffer U, Josting A, Franklin J, et al. NonHodgkin's lymphoma after primary Hodgkin's disease in the German Hodgkin's Lymphoma Study Group: incidence, treatment, and prognosis. J Clin Oncol 2001;19(7):2026.

12. Tucker MA, Coleman CN, Cox RS, et al. Risk of second cancers after treatment for Hodgkin's disease. N Engl J Med 1988;318(2):76.

13. Andreas Brauninger, Martin-Leo Hansmann, John G. Strickler et al.Identification of common Germinal Center B-cell precursors in two patientswith both Hodgkin's Disease and non-Hodgkin's lymphoma. N Engl J Med 1999;340:1239-1247.

14. Wickert RS, Weisenburger DD, Tierens A, et al. Clonal relationship between lymphocytepredominant Hodgkin's disease and concurrent or subsequent large-cell lymphoma of B lineage. Blood 1995; 86:2312-2320.

15. Greiner TC, Gascoyne RD, Anderson ME, et al. Nodular lymphocyte predominant Hodgkin's disease associated with large -cell lymphoma: Analysis of Ig gene rearrangements by V-J polymerase chain reaction. Blood 1996;88:657-666.

16. Zukerberg LR, Collins AB, Ferry JA, et al. Coexpression of CD15 andCD20 by Reed-Sternberg cells in Hodgkin's disease. Am J Pathol 1991;139: 475-483.

17. Chu WS, Abbondanzo SL, Frizzera G, et al. Inconsistency of the immunophenotype of ReedSternberg cells in simultaneous and consecutive specimens from the same patients: A paraffin section evaluation in 56patients. Am $\mathrm{J}$ Pathol 1992;141:11-17.

18. Enblad G, Sundstrom C, Glimelius B, et al. Immunohistochemical characteristics of Hodgkin and Reed-Sternberg cells in relation to age and clinical outcome. Histopathology 1993;22:535-541.

19. Bai MC, Jiwa NM, Horstman A, et al. Decreased expression of cellular markers in Epstein-Barr virus-positive Hodgkin's disease. J Pathol 1994;174:49-55.

20. Portlock CS, Donnelly GB, Qin J, et al. Adverse prognostic significance of CD20 positive ReedSternberg cells in classical Hodgkin's disease. Br J Haematol 2004;125:701-708.

21. Tzankov A, Zimpfer A, Pehrs AC, et al. Expression of B-cell markers in classical Hodgkin lymphoma: a tissue microarray analysis of 330 cases. Mod Pathol 2003;16:1141-1147.

22. Rassidakis GZ, Medeiros LJ, Viviani S, et al. CD2O Expression in Hodgkin and reed-sternberg cells of classical Hodgkin's disease: Associations with presenting features and clinical outcome. J Clin Oncol 2002;20:1278-1287.

23. Tzankov A, Krugmann J, Fend F, et al. Prognostic significance of CD20expression in classical Hodgkin lymphoma: A clinicopathological study of 119 cases. Clin Cancer Res 2003;9:1381-1386.

24. Swerdlow AJ, Douglas AJ, Hudson GV, et al. Risk of second primary cancers after Hodgkin's disease by type of treatment: analysis of 2846 patients in the British National Lymphoma Investigation. BMJ 1992;304(6835):1137.

25. Van Leeuwen FE, Klokman WJ, Hagenbeek A, et al. Second cancer risk following Hodgkin's disease: a 20-year follow-up study. J Clin Oncol 1994;12(2):312.

26. Mauch PM, Kalish LA, Marcus KC, et al. Second malignancies after treatment for laparotomy staged IA-IIIB Hodgkin's disease: long-term analysis of risk factors and outcome. Blood 1996;87(9):3625.

27. Abrahamsen JF, Andersen A, Hannisdal E, et al. Second malignancies after treatment of Hodgkin's disease: the influence of treatment, follow-up time, and age. J Clin Oncol 1993;11(2):255 . 
28. Swerdlow AJ, Douglas AJ, Vaughan Hudson G, et al. Risk of second primary cancer after Hodgkin's disease in patients in the British National Lymphoma Investigation: relationships to host factors, histology and stage of Hodgkin's disease, and splenectomy. Br J Cancer 1993;68(5):1006.

29. Bennett MH, MacLennan KA, Vaughan Hudson G, et al. Non-Hodgkin's lymphoma arising in patients treated for Hodgkin's disease in the BNLI: a 20-year experience. British National Lymphoma Investigation. Ann Oncol 1991;2 Suppl 2:83.

30. Zarate-Osorno A, Medeiros LJ, Longo DL, et al. Non-Hodgkin's lymphomas arising in patients successfully treated for Hodgkin's disease. A clinical, histologic, and immunophenotypic study of 14 cases. Am J Surg Pathol 1992;16(9):885.

31. Jacquillat C, Khayat D, Desprez-Curely JP, et al. Non-Hodgkin's lymphoma occurring after Hodgkin's disease. Four new cases and a review of the literature. Cancer 1984;53(3):459.

32. Pulte D, Gondos A, Brenner H. Long-term survival of patients diagnosed with non-Hodgkin lymphoma after a previous malignancy. Leuk Lymphoma 2009;50(2):179. 